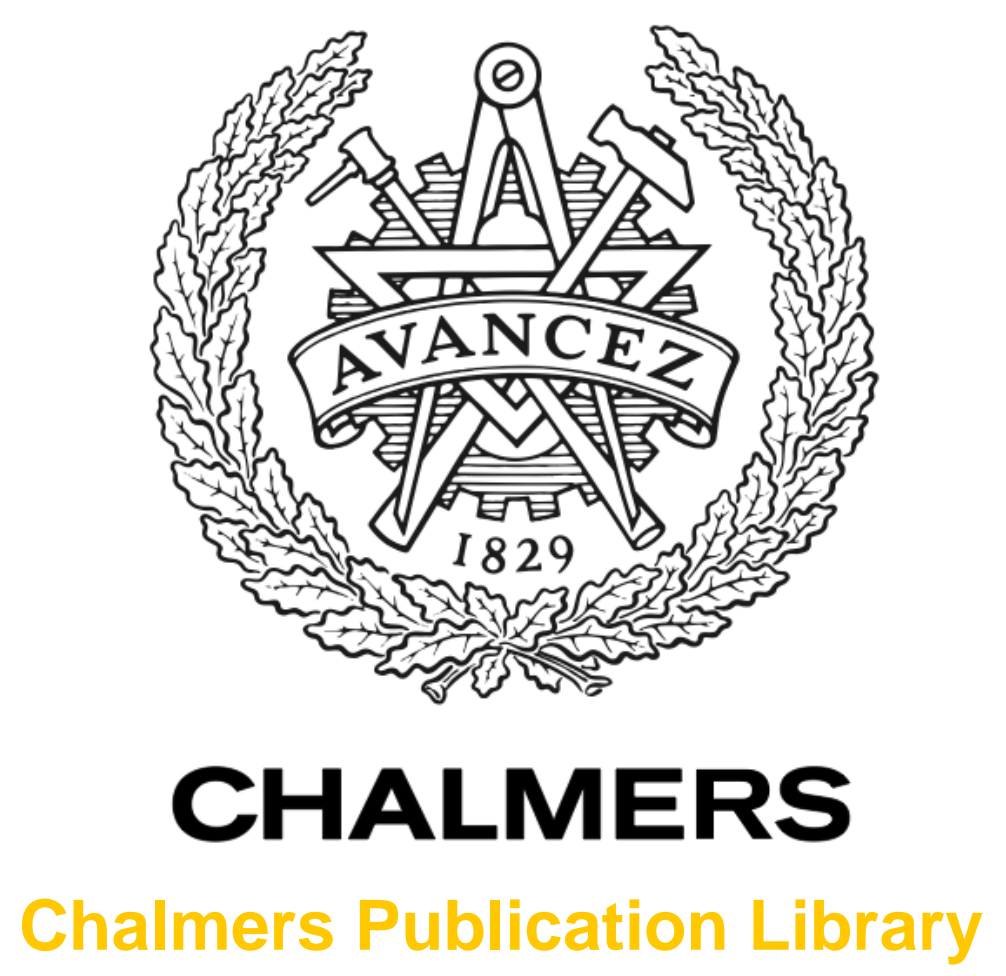

\title{
THz Josephson properties of grain boundary YBaCuO junctions on symmetric, tilted bicrystal sapphire substrates
}

This document has been downloaded from Chalmers Publication Library $(\mathrm{CPL})$. It is the author's version of a work that was accepted for publication in:

Journal of Applied Physics

Citation for the published paper:

Stepantsov, E. ; Tarasov, M. ; Kalaboukhov, A. (2004) "THz Josephson properties of grain boundary $\mathrm{YBaCuO}$ junctions on symmetric, tilted bicrystal sapphire substrates". Journal of Applied Physics, vol. 96(6), pp. 3357-3361.

Downloaded from: http://publications.lib.chalmers.se/publication/2028

Notice: Changes introduced as a result of publishing processes such as copy-editing and formatting may not be reflected in this document. For a definitive version of this work, please refer to the published source. Please note that access to the published version might require a subscription. 


\title{
THz Josephson properties of grain boundary YBaCuO junctions on symmetric, tilted bicrystal sapphire substrates
}

\author{
E. Stepantsov \\ Institute of Crystallography RAS, 117333, Moscow, Russia \\ M. Tarasov \\ Institute of Radio Engineering and Electronics RAS, 125009, Moscow, Russia
}

A. Kalabukhov, L. Kuzmin, and T. Claeson

Chalmers University of Technology, SE 41296, Gothenburg, Sweden

(Received 9 March 2004; accepted 18 June 2004)

\begin{abstract}
Superconducting Josephson junctions with high characteristic voltages $\left(I_{c} R_{n}\right.$ larger than $4 \mathrm{mV}$ at 4.2 $\mathrm{K})$ are fabricated by depositing $\mathrm{YBa}_{2} \mathrm{Cu}_{3} \mathrm{O}_{7-x}$ on tilted sapphire bicrystal substrates, where the tilting axis is along the grain boundary. The good junction quality and low microwave losses in sapphire gave high frequency response well into the THz region. High quality $\mathrm{YBa}_{2} \mathrm{Cu}_{3} \mathrm{O}_{7-x}$ epitaxial films were deposited on tilted sapphire substrates with $\mathrm{CeO}_{2}$ buffer layers by pulsed laser deposition. $\mathrm{YBaCuO}$ films have smaller tilt angles, from $1.0^{\circ}$ up to $10.3^{\circ}$, compared to inclination angles of the substrates from $1.5^{\circ}$ to $13.6^{\circ}$. X-ray diffraction shows only a single orientation of the films in the $a$ - $b$ plane, as well as an absence of $a$-axis particles and outgrowths. Critical temperatures as high as $T_{c}=88.5-89.0 \mathrm{~K}$ and $\Delta T_{c} \leqslant 1.5 \mathrm{~K}$ were obtained in all films. The grain boundary in a common high- $T_{c}$ superconducting junction is usually much less straight than in the in-plane rotated bicrystal substrate, depressing Josephson currents. The waviness of the artificial grain boundary in a tilted bicrystal film is three times less than in an in-plane (untilted) bicrystal. Tilted Josephson junctions of widths from 1.5 to $6 \mu \mathrm{m}$ were tested at temperatures from 0.26 to $77 \mathrm{~K} . I_{c} R_{n}$ products as high as $4.5 \mathrm{mV}$ were observed at $T=4.2 \mathrm{~K}$. Shapiro steps were observed at voltages over $3 \mathrm{mV}$ under $300 \mathrm{GHz}$ irradiation. Josephson radiation from the tilted junction was measured at frequencies up to $1.7 \mathrm{THz}$ by a cryogenic bolometer. Suppressing the critical current with a magnetic field can separate Josephson radiation and thermal radiation. A parabolic dependence of the response on bias voltage for thermal radiation corresponds to an increase of junction temperature from $260 \mathrm{mK}$ at zero bias to $3 \mathrm{~K}$ at $1 \mathrm{mV}$ bias. (C) 2004 American Institute of Physics. [DOI: 10.1063/1.1782273]
\end{abstract}

\section{INTRODUCTION}

The development of terahertz $(\mathrm{THz})$ frequency receivers for radio astronomy, remote atmosphere monitoring, biomedical research, or communication technology requires new ultrasensitive detectors, high-resolution spectrometers, and broadband tunable oscillators. Conventional semiconducting detectors are limited in sensitivity and bandwidth. Fourier transform spectrometers, backward wave oscillators, and far infrared lasers are usually big, heavy, expensive, and complicated in operation. They cannot be considered as air or space borne due to high weight, high power consumption, and mechanical instability. An alternative is to use superconducting electronic devices such as Josephson direct detectors, superconductor-insulator-superconductor mixers, Hilbert transform spectrometers, Josephson oscillators, or superconducting quantum interference devices sensors and amplifiers. Contrary to conventional low critical temperature devices based on $\mathrm{Nb}$ and $\mathrm{NbN}$ technology, with the natural frequency limit set to below $1 \mathrm{THz}$ by the superconducting energy gap, high critical temperature superconductors (HTS) potentially lift this frequency limit to over $10 \mathrm{THz}$. High critical temperature bicrystal Josephson junctions are promising candidates for $\mathrm{THz}$ band detectors, oscillators, and Hilbert spectrometers. The technology of bicrystal junctions is the most developed one for HTS Josephson junctions, and it has the best performance compared to other technologies.

A conventional grain boundary Josephson junction is epitaxially grown on a bicrystal substrate with an in-plane misorientation angle. Further improvement of film and junction characteristics can be obtained by using tilted substrates. In this case the film growth changes from island or spiral growth to step flow growth leading to less surface roughness and an increase of current density. ${ }^{1,2}$ Transport properties become more anisotropic for tilt angles over $5^{\circ} .^{2-4}$ Conventional in-plane bicrystal junctions are well described in several published works and are widely used in superconducting devices. ${ }^{5-7}$ Other types of bicrystal junctions are less frequently treated. ${ }^{5,8}$ It was recently demonstrated ${ }^{9,10}$ that tilted grain boundary junctions can have much better performance margins than those with in-plane misorientation. The tilted film grain boundary is straight and follows the bicrystal substrate boundary. It shows a low degree of waviness in comparison to that of a conventional in-plane grain boundary. The work by Divin et al. ${ }^{10}$ demonstrated excellent dc characteristics for $\mathrm{YBaCuO}$ Josephson junctions on [100]-tilt $\mathrm{SrTiO}_{3}$ bicrystals. An $I_{c} R_{n}$ product of up to $1.2 \mathrm{mV}$ at $77 \mathrm{~K}$ and $8 \mathrm{mV}$ at $4.2 \mathrm{~K}$ shows a potential of this technology at $\mathrm{THz}$ frequencies. Nevertheless, high microwave losses in $\mathrm{SrTiO}_{3}$ do not allow all advantages of a high $I_{c} R_{n}$ product to 
be obtained and Shapiro steps were observed only up to 94 GHz. To reduce microwave losses and improve the impedance matching, $\mathrm{NdGaO}_{3}$ tilted bicrystal junctions were developed. ${ }^{11}$ High-quality [001]-tilt $\mathrm{YBaCuO}$ grainboundary junctions were fabricated on untwinned 2 $\times 14^{\circ}(110) \mathrm{NdGaO}_{3}$ bicrystal substrates for applications at $\mathrm{THz}$ frequency. $I_{c} R_{n}$ approaches $1.8 \mathrm{mV}$ in such junctions, the maximum of the response was observed at a signal frequency of $1.19 \mathrm{THz}$, and clear detector response was observed up to $3.1 \mathrm{THz}$. To increase the frequency margin further, we decided to use sapphire tilted bicrystal substrates. Microwave losses in sapphire are low, thermal conductivity is high.

\section{FILM DEPOSITION, CHARACTERIZATION, AND JUNCTION PATTERNING}

Epitaxial $\mathrm{YBaCuO}$ films were grown by pulsed laser deposition on $\mathrm{Al}_{2} \mathrm{O}_{3}$ and $\mathrm{Y}-\mathrm{ZrO}_{2}$ monocrystal substrates that were tilted from a standard orientation of (1012) and (001) by $2^{\circ}-14^{\circ}$. Sapphire symmetric bicrystal substrates with the same tilt of the monocrystal parts towards the boundary direction were used to fabricate Josephson junctions. Thicknesses of all films are $250 \mathrm{~nm}$. The tilt of the $c$ axis in one batch of films was in the $\langle 100\rangle$ direction and in the $\langle 110\rangle$ direction in another batch. A buffer layer of $\mathrm{CeO}_{2}$ was deposited at $770{ }^{\circ} \mathrm{C}$ in an oxygen atmosphere of 0.3 mbar prior to the $\mathrm{YBaCuO}$ film deposition. $\mathrm{A} \mathrm{YBaCuO}$ film was deposited in the same vacuum cycle at $780{ }^{\circ} \mathrm{C}$ and oxygen pressure 0.6 mbar. An in situ Au film was deposited afterwards. A thick layer of $\mathrm{Au}$ was then deposited by thermal evaporation for a planar antenna and contact pads. The deposited films were patterned by deep ultraviolet (UV) lithography and ion beam etching in an Ar plasma to form Josephson junctions, planar antennas and contact pads.

The crystalline structure of the films was investigated in an X-ray diffractometer "Philips X'prt MRD" in $\Theta / 2 \Theta$ and $\phi$-scan modes using an $\mathrm{x}$-ray lens and a slot collimator. A $\Theta / 2 \Theta$-scan of a $\mathrm{YBaCuO}$ film deposited on a sapphire single crystal substrate with a tilt angle of the surface related to the (1012) plane by $14^{\circ}$ to the direction [2201] is presented in Fig. 1. It corresponds to the direction $\langle 100\rangle$ in the $\mathrm{YBaCuO}$ film. By this measurement, the exact offset of $\Theta$ was determined for the $\mathrm{YBaCuO}$ film, the $\mathrm{CeO}_{2}$ buffer layer, and the substrate, corresponding to the tilt angles of their crystalline lattices relatively the surface of the film. It was found that this offset is different for the buffer and the $\mathrm{YBaCuO}$ films. The $(\Theta / 2 \Theta)$-scan curve in Fig. 1 corresponds to the offset of the $\mathrm{YBaCuO}$ film. One can see that there are no additional peaks that can be due to $\mathrm{CuO}$ and $\alpha$ particles. A $\phi$-scan, corresponding to the (116) peak and covering $150^{\circ}$, shows an absence of peaks due to other orientations of $\mathrm{YBaCuO}$ in the $a-b$ plane. It means that the film is single domain. In the $(\Theta / 2 \Theta)$-scan curve, there are practically no substrate peaks that are determined by nonparallel orientation with deflection up to $3.5^{\circ}$ between crystal lattices of film and substrate. Tilt angles of film and buffer layers are always less than the substrate angle. For substrates with lower angles, we ob-

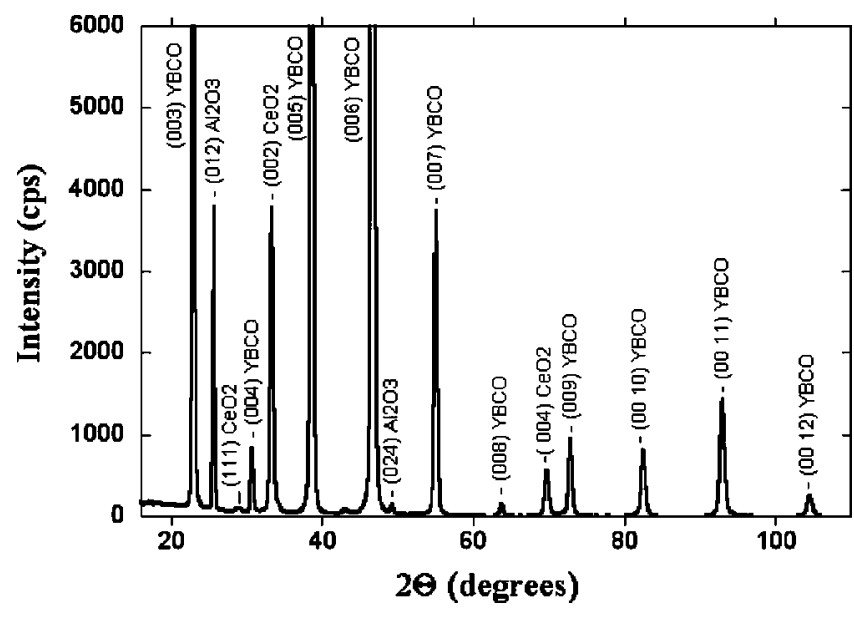

FIG. 1. XRD $\theta / 2 \theta$-scan of a YBCO $250 \mathrm{~nm}$ thick film grown on a $\mathrm{CeO}_{2}$ buffered sapphire substrate with a $14^{\circ}$ tilt of the normal to a (1012) plane relatively the normal to the surface in direction [2201]. This direction corresponds to $\langle 100\rangle \mathrm{YBaCuO}$.

served a similar difference. Figure 2 displays the difference in tilt angles between the film and the substrate versus the tilt angle of the substrate.

Surfaces of the films were examined by a scanning probe microscope (SPM). A surface of a $\mathrm{YBaCuO}$ film tilted by $11^{\circ}$ on a sapphire substrate tilted by $14^{\circ}$ is shown in Fig. 3. One can see that there are subgrains at the surface and they are elongated in the $a-b$ plane in a direction perpendicular to the tilt direction. The profile of this surface in the direction of the tilt shows a valley depth about 11-14 $\mathrm{nm}$ and the average roughness $R_{a}$ does not exceed $1.4 \mathrm{~nm}$. These values correspond to a film thickness of $250 \mathrm{~nm}$. The SPM profile in the vicinity of the bicrystal boundary (Fig. 4) shows a V-shaped surface suppression of about $40 \mathrm{~nm}$ depth. This boundary is much straighter than those of conventional nontilted bicrystal films. The average length of subgrains along the boundary is 3-5 times longer than width, contrary to the grains in conventional bicrystals with equal length and width. All our SPM data confirm that the roughness of a $250 \mathrm{~nm}$ thick

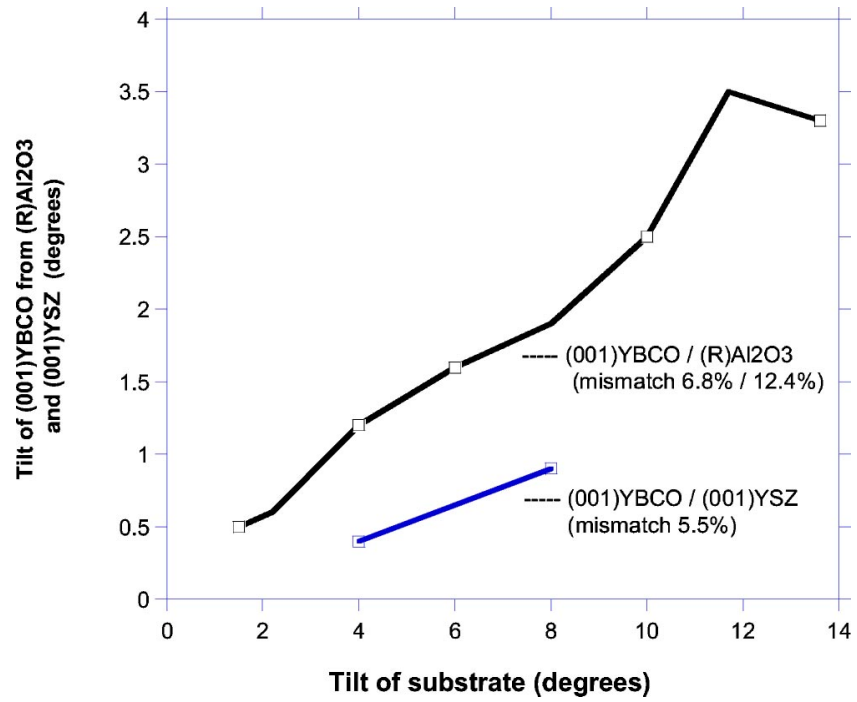

FIG. 2. The substrate tilt dependence of $\mathrm{YBaCuO}$ film lattice inclination relatively substrate (sapphire and YSZ) crystalline lattice. 


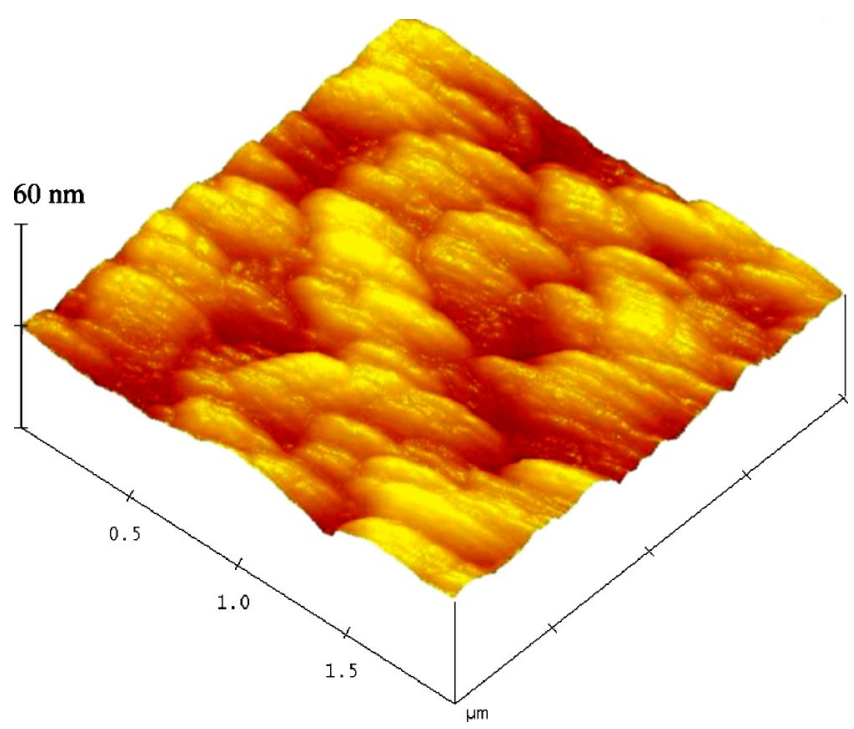

FIG. 3. (Color online) Three-dimensional SPM image of a YBaCuO thin film grown on a sapphire substrate with a $14^{\circ}$-tilt of the normal to the (1012) plane relatively the normal to the surface in direction [2201].

$\mathrm{YBaCuO}$ tilted film on sapphire is 1.5 times less than those of $100 \mathrm{~nm}$ thick films on tilted $\mathrm{SrTiO}_{3}$ and $\mathrm{NdGaO}_{3}$ substrates. ${ }^{9,10}$ The roughness increases with increasing thickness and the difference in roughness should be even higher in favor of our films on sapphire if equal film thicknesses were considered. We can conclude that a low roughness adds to the advantages of tilted sapphire substrates.

\section{CHARACTERIZATION OF JOSEPHSON JUNCTIONS}

"Valley"-type $2 \times 11^{\circ}[100]$-tilt grain boundary junctions of width from 1.5 to $6 \mu \mathrm{m}$, which were fabricated on 2 $\times 14^{\circ}[100]$-tilt sapphire bicrystals, demonstrated resistively shunted junction-type IV curves in a wide range of temperatures from $260 \mathrm{mK}$ to $77 \mathrm{~K}$. The critical current density was about $1500 \mathrm{kA} / \mathrm{cm}^{2}$ at $4.2 \mathrm{~K}$ and $20-40 \mathrm{kA} / \mathrm{cm}^{2}$ at $77 \mathrm{~K}$. An $I_{c} R_{n}$-product of up to $4.5 \mathrm{mV}$ was observed at $4.2 \mathrm{~K}$ and up to $150 \mu \mathrm{V}$ at $77 \mathrm{~K}$. This is much higher compared to conventional $a-b$ junctions on in-plane sapphire bicrystals. The excess current did not exceed $20 \%$ of the maximum

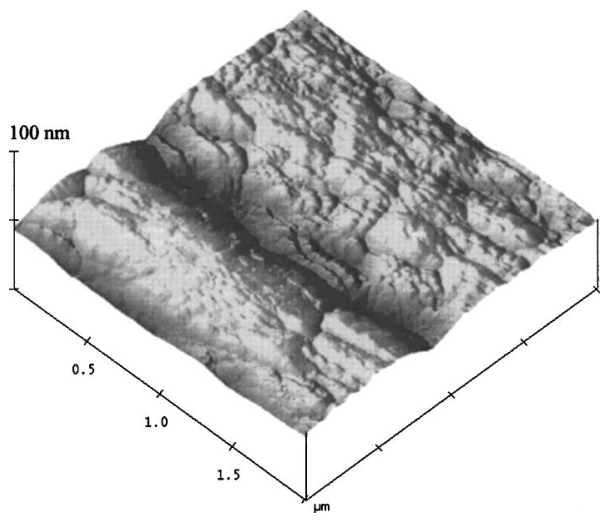

FIG. 4. Three-dimensional SPM picture of a YBaCuO thin film grown on a sapphire symmetric bicrystal substrate. In both single crystalline parts the (1012) planes are tilted by $14^{\circ}$ relatively to the surface in directions [2201] to the grain boundary.

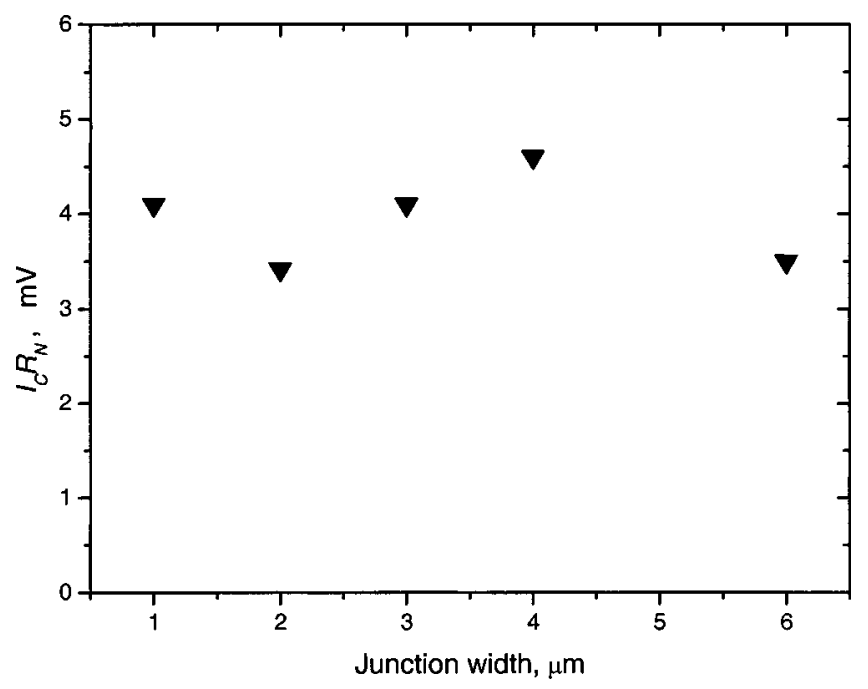

FIG. 5. Dependence of the $I_{C} R_{N}$ product at $T=4.2 \mathrm{~K}$ on the width of 2 $\times 14^{\circ}$ [100]-tilt grain boundary $\mathrm{YBaCuO}$ junctions fabricated on sapphire bicrystal substrates.

critical current at $77 \mathrm{~K}$ and did not substantially change with temperature. The dependence of the $I_{C} R_{N}$ value on the junction width is shown in Fig. 5. It was $4 \pm 0.5 \mathrm{mV}$ and no prominent width dependence was noted.

The Josephson oscillator chip consists of five junctions integrated with planar antennas. The log-periodic antenna in the oscillator chip is designed for frequencies 100-1000 $\mathrm{GHz}$. Up to five Shapiro steps were observed in IV curves at $T=4.2 \mathrm{~K}$ under radiation at $300 \mathrm{GHz}$ (see Fig. 6). Critical current and Shapiro steps oscillate with applied microwave power. The case of zero critical current and maximal amplitude of the first step is presented in Fig. 6.

Josephson radiation was measured in two ways. A cryogenic bolometer ${ }^{12}$ was used as a detector in both cases. In the first experiment its chip was attached to the substrate with the Josephson junction and cooled down to $260 \mathrm{mK}$. The critical current of the bicrystal junction varied from 20 to 2

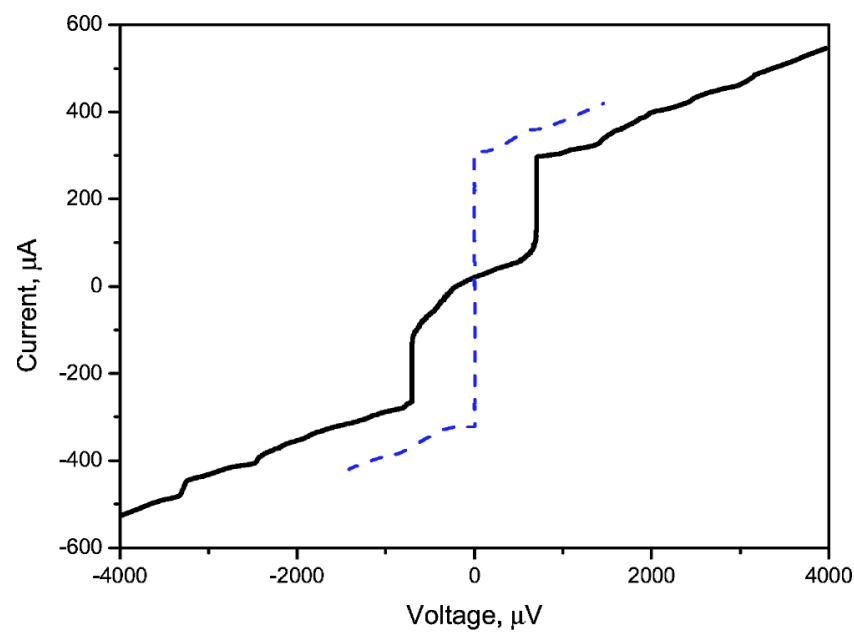

FIG. 6. IV-curve of a "valley"-type $2 \times 14^{\circ}[100]$-tilt grain boundary junction at $4.2 \mathrm{~K}$ under $322 \mathrm{GHz}$ irradiation. The critical current is completely suppressed by the microwave radiation, and the first Shapiro step has the largest amplitude. The fifth step is seen at $3.2 \mathrm{mV}$. The dashed line is the IV-curve without radiation. 


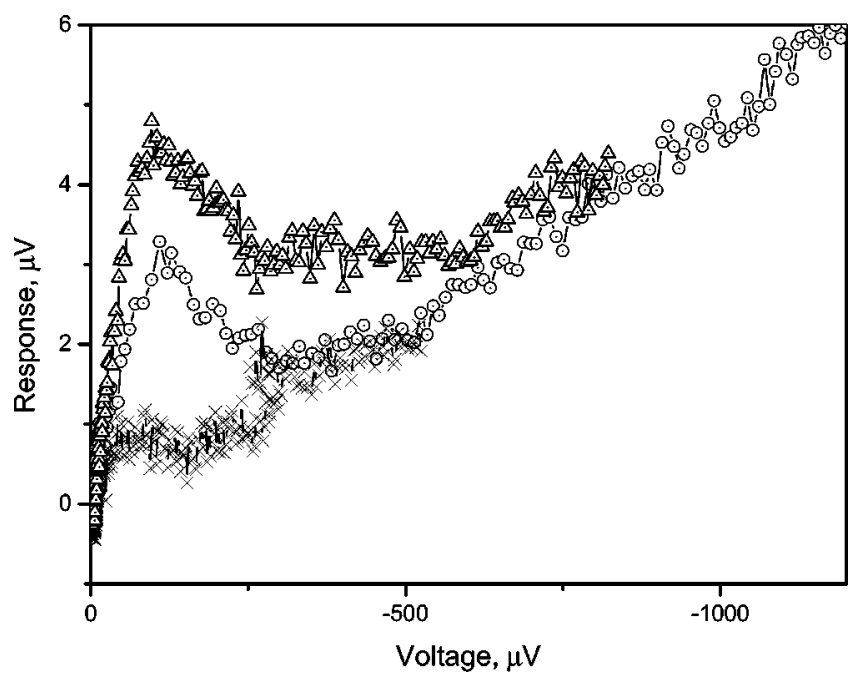

FIG. 7. Detected response of the bolometer as a function of voltage in a $\mathrm{YBaCuO}$ Josephson oscillator and three different levels of magnetic field. The substrate temperature is about $260 \mathrm{mK}$.

$\mu \mathrm{A}$ at different values of magnetic field produced by a permanent magnet placed $10 \mathrm{~cm}$ outside the cryostat. The detected response (Figs. 7 and 8) consists of two components. The first one is independent of magnetic field and corresponds to infrared radiation from an overheated Josephson junction. The second component is largest for the highest critical current and can be suppressed by a magnetic field. By suppressing the critical current of the Josephson junction, it is possible not only to decrease the output power of the oscillations, but also to reduce their frequency range. When the critical current is suppressed below $2 \mu \mathrm{A}$ the response voltage is clearly proportional to the square of the JJ bias current. This means that we can separate Josephson radiation at frequencies below $1 \mathrm{THz}$ and infrared radiation of an overheated junction for bias voltages over $1 \mathrm{mV}$. For Josephson oscillations we can roughly estimate the maximum available power in a matched load and at the characteristic frequency as $P_{\text {osc }} \leqslant 0.1 \times I_{c} V_{c}=2 \times 10^{-9} \mathrm{~W} .{ }^{13}$ Misalignment of antennas, mismatch of beam patterns, polarization, and mismatch of impedances can bring the total attenuation of radiation

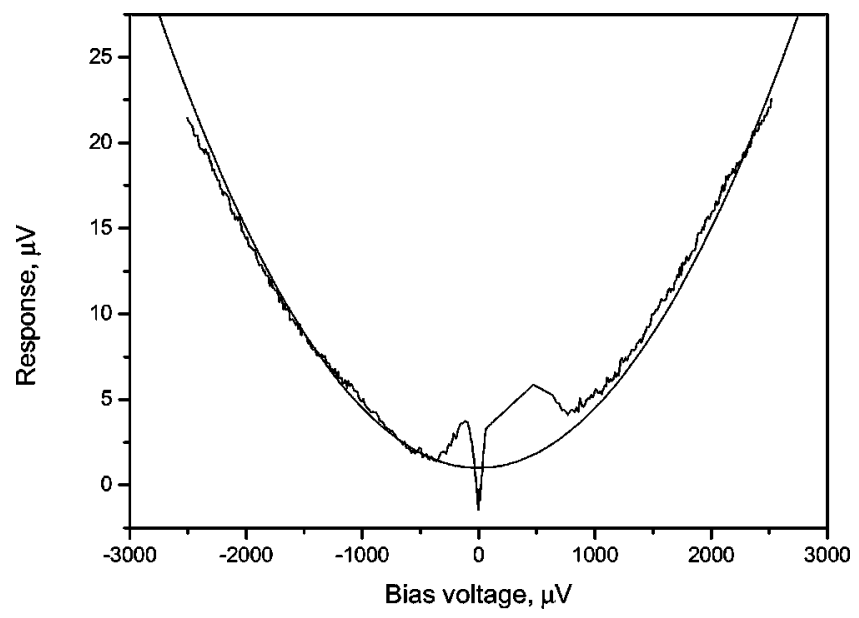

FIG. 8. Response within a wide bias voltage range across the same Josephson junction, and a fitting parabola (smooth line) given by Eq. (4) The initial temperature is $260 \mathrm{mK}$ but depends on bias voltage. power up to $30 \mathrm{~dB}$. That would correspond to an available power at the bolometer of about $10^{-12} \mathrm{~W}$. The estimated bolometer responsivity is $S=1.1 \times 10^{8} \mathrm{~V} / \mathrm{W}$, which brings the maximum voltage response at this power to about $10^{-4} \mathrm{~V}$. We measured voltage responses of up to $10 \mu \mathrm{V}$ in our experiments. The order of magnitude difference in response can be explained by a nonideal behavior of the Josephson junction (excess current) and overheating that reduce output power. The response to non-Josephson radiation at the parabolic part of the response curve can be attributed to submillimeter and IR radiation from a matched overheated load placed in the center of the antenna. We approximate the overheating in a JJ by a model given in Ref. 14 for a variable thickness microbridge as follows:

$$
T_{m}=\sqrt{T_{b}^{2}+3\left(\frac{e V}{2 \pi k}\right)^{2}},
$$

where $T_{b}$ is the bath temperature $(260 \mathrm{mK})$. It results in an equivalent electron temperature of about $3 \mathrm{~K}$ at $1 \mathrm{mV}$ bias. Taking into account that the radiation is spread in a $4 \pi$ solid angle and the bolometer (at a distance of over $1 \mathrm{~mm}$ through a dielectric) can absorb only a small part of this radiation, the measured increase in temperature of $5 \mathrm{mK}$ looks reasonable and corresponds to losses of about $30 \mathrm{~dB}$ between oscillator and bolometer. Bolometer response was separately calibrated by varying the cold stage temperature. We should now take into account that this power is radiated and then received. It means that Planck's radiation law should be applied

$$
P_{\mathrm{rad}}=\frac{h f}{\exp \left(\frac{h f}{k T}\right)-1} \Delta f .
$$

For our experiment we can take the band width of the double dipole antenna $\Delta f=0.3 f$. At relatively low frequencies the power can be reduced to $k T \Delta f$ and at high frequencies it reduces to $h f \Delta f \exp (-h f / k T)$. If we apply the Planck formula and use Eq. (1), neglecting the phonon temperature,

$$
P_{\text {rad }}=\frac{0.3 h f^{2}}{\exp \left(\frac{h f}{0.27 e V}\right)-1},
$$

which is square-law dependent at low bias and linear at high bias voltage (over $10 \mathrm{mV}$ ). For our design frequency $f$ $=300 \mathrm{GHz}$, frequency corresponds to the bias voltage as $f$ $=2 \mathrm{eV} / \mathrm{h}$, and in the bias range up to $5 \mathrm{mV}$, Eq. (3) can be roughly fitted with the approximate expression

$$
P_{\mathrm{rad}}=\frac{0.6}{4 \pi^{2}} \frac{e^{2} V^{2}}{h} \text {. }
$$

This approximation is presented in Fig. 8 as a fitting parabola. It shows the right parabolic voltage dependence of the measured power.

In the second experiment with high critical current junctions, $I_{c}>0.5 \mathrm{~mA}$, it was necessary to place the Josephson oscillator separately from the bolometer to avoid overheating. This was arranged in a quasioptical configuration where the substrate with the Josephson junction was attached to a sapphire hyperhemisphere lens at the $1.8 \mathrm{~K}$ stage and the 


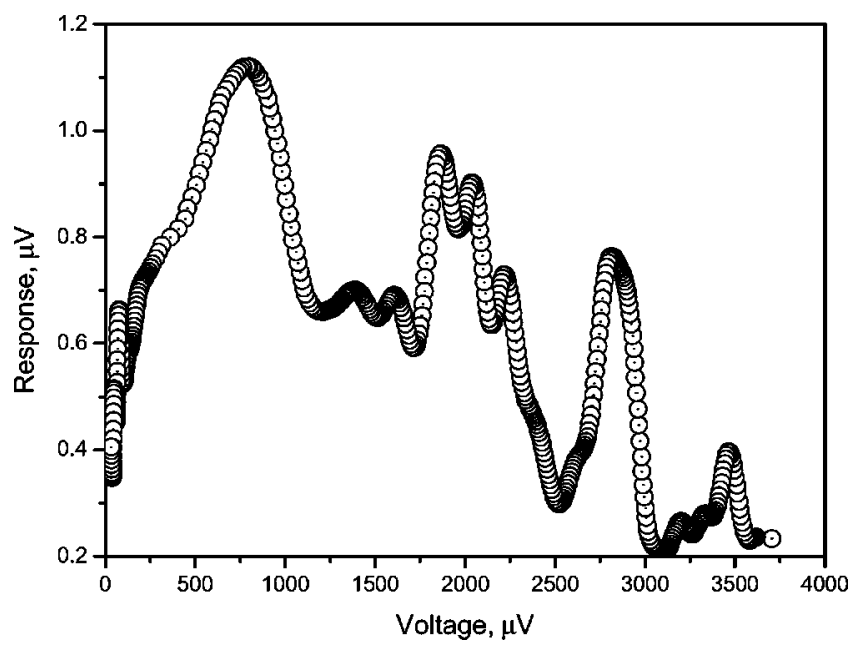

FIG. 9. The bolometer response versus bias voltage of the $2 \times 14^{\circ}$ [100]-tilt grain boundary $\mathrm{YBaCuO}$ Josephson oscillator. The temperature of the oscillator was $1.8 \mathrm{~K}$, of the bolometer $0.26 \mathrm{~K}$.

bolometer with its Si lens was placed at the $260 \mathrm{mK}$ stage with lenses facing each other. The results presented in Fig. 9 show signal maxima at voltages $0.8,1.9,2.8$, and $3.5 \mathrm{mV}$ that correspond to the spectral dependencies of the antennas and the quasioptical beamguide. The signal maximum at 3.5 $\mathrm{mV}$ corresponds to frequency $1.7 \mathrm{THz}$. We can suppress all these maxima by a magnetic field, which proves that Josephson radiation is the origin of the received signal.

\section{DISCUSSION}

An important result of our investigation of epitaxial film growth on tilted sapphire crystals is that the tilt angle of the film is different from the tilt angle of the crystal lattice of the substrate. The difference in lattice tilt angles relative to the surface can be explained by the thermodynamic tendency to minimize the surface energy of the film by tilting it towards the surface of a low-index plane.

The high value of the $I_{c} R_{n}$-product, and its small spread, for a symmetric bicrystal junction can be explained by a more regular boundary in a [100]-tilt grain boundary compared to a conventional [001]-tilt grain boundary, at which a significant faceting exists. As mentioned by Mannhart, Tafuri and their co-workers, ${ }^{8,15}$ the suppression of the order parameter is not as high in the case of a [100]-tilt grain boundary as in an ordinary [001]-tilt boundary due to the specific symmetry. This is a reasonable explanation of the higher $I_{c} R_{n}$-product for a [100]-tilt grain boundary.

Direct heating of HTS Josephson junctions at bias voltages over $1 \mathrm{mV}$ can be an important limiting factor for terahertz applications. The bias voltage is about $10 \mathrm{mV}$ for a 5 $\mathrm{THz}$ signal and overheating can go up to $30 \mathrm{~K}$. The actual critical current for such a bias voltage will be the same as measured at $30 \mathrm{~K}$. This is much less than at $4 \mathrm{~K}$, the $I_{c} R_{n}$ product will be reduced proportionally and, as a result, the high-frequency response will be suppressed. To reduce such overheating, the heat sink should be improved by using a substrate with a high thermal conductivity and through a thick-film, normal metal quasiparticle trap (gold in our case) deposited on top of $\mathrm{YBaCuO}$ and which is also used as a planar antenna.

\section{CONCLUSION}

We have fabricated high-quality epitaxial $\mathrm{YBaCuO}$ films and bicrystal junctions on tilted $\mathrm{Al}_{2} \mathrm{O}_{3}$ substrates, which have low dielectric losses. Such junctions on symmetric vicinal sapphire bicrystals with [100]-tilt showed an $I_{c} R_{n}$-product over $4 \mathrm{mV}$ and a small spread of characteristics. Josephson radiation up to $1.7 \mathrm{THz}$ was directly measured by a cryogenic bolometer. A high characteristic frequency of about $2 \mathrm{THz}$ and low microwave losses in sapphire make tilted junctions promising candidates for terahertz applications such as a direct Josephson detector, a Josephson oscillator, or a Hilbert spectrometer. It can also be used as a $\mathrm{THz}$ band cryogenic network analyzer in combination with a cryogenic bolometer.

\section{ACKNOWLEDGMENTS}

This work was supported in part by the Swedish Research Council (VR), Royal Academy of Sciences (KVA), Foundation of Strategic Research (SSF), STINT, the Swedish Institute, and by INTAS-01-686.

${ }^{1}$ L. Mechin, P. Berghus, and J. Evetts, Physica C 302, 102 (1998).

${ }^{2}$ P. Czerwinka, R. Campion, K. Horbelt, P. King, S. Misat, S. Morley, H. Habermeier, and B. Leibold, Physica C 324, 96 (1999).

${ }^{3}$ J. Pedarnig, R. Rossler, M. Deamare, W. Lang, and D. Bauerle, Appl. Phys. Lett. 81, 2587 (2002).

${ }^{4}$ Y. Divin, U. Poppe, J. Seo, B. Kabius, and K. Urban, Physica C 235-240, 675 (1994).

${ }^{5}$ D. Dimos, P. Chaudhari, and J. Mannhart, Phys. Rev. B 41, 4038 (1990).

${ }^{6}$ Z. G. Ivanov, P. A. Nilsson, D. Winkler, J. A. Alarco, T. Claeson, E. A. Stepantsov, and A. Ya. Tzalenchuk, Appl. Phys. Lett. 59, 3030 (1991).

${ }^{7}$ M. Tarasov et al., Supercond. Sci. Technol. 12, 995 (1999).

${ }^{8}$ F. Tafuri, F. Graozio, F. Carillo, F. Lombardi, K. Verbist, O. Lebedev, and G. van Tendeloo, Physica C 326-327, 63 (1999).

${ }^{9}$ U. Poppe, Y. Divin, M. Faley, J. Wu, C. Jia, P. Shadrin, and K. Urban, IEEE Trans. Appl. Supercond. 11, 3768 (2001).

${ }^{10}$ Y. Divin, U. Poppe, C. Jia, P. Shadrin, and K. Urban, Physica C 372-376, 115 (2002).

${ }^{11}$ Y. Divin, O. Volkov, M. Liatti, and V. Gubankov, IEEE Trans. Appl. Supercond. 13, 676 (2003)

${ }^{12}$ M. Tarasov, M. Fominsky, A. Kalabukhov, and L. Kuzmin, JETP Lett. 76, 507 (2002)

${ }^{13} \mathrm{~K}$. Likharev, Dynamics of Josephson junctions and circuits (Gordon and Breach, Amsterdam, 1986).

${ }^{14}$ M. Tinkham, M. Octavio, and W. J. Skocpol, J. Appl. Phys. 48, 1311 (1977).

${ }^{15}$ J. Mannhart and H. Hilgenkamp, Supercond. Sci. Technol. 10, 880 (1997). 